TIFR/TH/05-40

\title{
Dynamics of Fluctuation Dominated Phase Ordering: Hard-core Passive Sliders on a Fluctuating Surface
}

\author{
Sakuntala Chatterjee and Mustansir Barma \\ Department of Theoretical Physics, Tata Institute of Fundamental Research, Homi Bhabha Road, Mumbai-400005, India
}

\begin{abstract}
We study the dynamics of a system of hard-core particles sliding downwards on a one dimensional fluctuating interface, which in a special case can be mapped to the problem of a passive scalar advected by a Burgers fluid. Driven by the surface fluctuations, the particles show a tendency to cluster, but the hard-core interaction prevents collapse. We use numerical simulations to measure the auto-correlation function in steady state and in the aging regime, and space-time correlation functions in steady state. We have also calculated these quantities analytically in a related surface model. The steady state auto-correlation is a scaling function of $t / L^{z}$, where $L$ is the system size and $z$ the dynamic exponent. Starting from a finite intercept, the scaling function decays with a cusp, in the small argument limit. The finite value of the intercept indicates the existence of long range order in the system. The space-time correlation, which is a function of $r / L$ and $t / L^{z}$, is non-monotonic in $t$ for fixed $r$. The aging auto-correlation is a scaling function of $t_{1}$ and $t_{2}$ where $t_{1}$ is the waiting time and $t_{2}$ the time difference. This scaling function decays as a power law for $t_{2} \gg t_{1}$; for $t_{1} \gg t_{2}$, it decays with a cusp as in steady state. To reconcile the occurrence of strong fluctuations in the steady state with the fact of an ordered state, we measured the distribution function of the length of the largest cluster. This shows that fluctuations never destroy ordering, but rather the system meanders from one ordered configuration to another on a relatively rapid time scale.
\end{abstract}

\section{INTRODUCTION}

The concentration of a substance advected by a driving field such as a fluid flow often shows interesting behavior. In examples such as smoke dispersed in air or fluorescent dye carried by a turbulent jet, an initial local concentration of the passive particles generally spreads out in space 1]. However, if the fluid is compressible, instead of spreading out, the advected substance may show a tendency to cluster, as with air bubbles in water or dust particles in air 2, 3].

In the situations discussed above, the advected substance has a negligible effect on the fluid flow. These problems are examples of 'passive scalar' problems, where the dynamics of a non-equilibrium driving field strongly affects that of the other (passive) field with no back-effect from the latter. In this paper, we study clustering in a set of passive particles which are subjected to a fluctuating force field. The specific system we study consists of hard-core particles sliding under gravity on a one dimensional fluctuating interface; the instantaneous force on a particle is then proportional to the local slope of the surface. Interestingly, our results also pertain to particles advected by a fluid, using the fact that the equation governing a moving interface can be mapped onto the Burgers equation, which describes a compressible fluid [4].

The degree of clustering of the particles depends strongly on the interactions between them. Two cases have been studied earlier - particles which are completely non-interacting [5, 6] and particles which interact via hard-core exclusion [7, 8]. We study the latter, more realistic, case in this paper and address two broad issues, namely dynamics and ordering.

The first issue concerns the time-dependent properties of the passive particles. We obtain results for the auto-correlation and space-time correlation functions in steady state, and aging correlations in the approach to steady state. To our knowledge, the dynamics of passive scalars has not been explored systematically, and our study adds to the relatively sparse work on this important question [9]. We have considered two kinds of surface evolutions - namely those that respect symmetry under reflection (Edwards-Wilkinson type) and those which break this symmetry (Kardar-Parisi-Zhang type). Our simulation results for the dynamics of the sliding particle system are supplemented by analytical calculations for a related model of coarse-grained interface variables.

The second issue concerns the characterization of the steady state as an ordered state. Earlier studies of static properties revealed that strong fluctuations are present in the steady state and do not decrease even in the thermodynamic limit 7]. On the other hand, the scaling function for the density correlation indicates that the system has long range order. This sort of fluctuation dominated phase ordering (FDPO) is characterized by a broad distribution of the order parameter. The question then arises: In what sense does FDPO represent an ordered state, if strong fluctuations drive it between macroscopically different configurations on a relatively rapid time-scale? We address this by studying the variation of the length of the largest particle cluster present in the system and show that the corresponding probability distribution provides an unequivocal signal of ordering. 


\section{OVERVIEW}

\section{A. Surface Fluctuation and Particle Movement}

A surface with no overhangs is completely specified by the height $h(x, t)$ at point $x$ at time $t$. The evolution of the height field is taken to be described by the KardarParisi-Zhang (KPZ) equation [4].

$$
\frac{\partial h}{\partial t}=\nu_{1} \frac{\partial^{2} h}{\partial x^{2}}+\lambda\left(\frac{\partial h}{\partial x}\right)^{2}+\eta_{1}(x, t)
$$

The first term represents the smoothening effect of surface tension $\nu_{1}$, and $\eta_{1}(x, t)$ is a white noise with zero average and $\left\langle\eta_{1}(x, t) \eta_{1}\left(x^{\prime}, t^{\prime}\right)\right\rangle=\Gamma \delta\left(x-x^{\prime}\right) \delta\left(t-t^{\prime}\right)$. Notice that if $\lambda \neq 0, h \rightarrow-h$ symmetry is not preserved, reflecting the fact that the interface moves in a preferred direction. However, if $\lambda=0$, the equation has an $h \rightarrow-h$ symmetry and describes the Edwards-Wilkinson (EW) model 10 .

The height-height correlation function has a scaling form for large separations in space and time [11] :

$$
\left\langle\left[h(x, t)-h\left(x^{\prime}, t^{\prime}\right)\right]^{2}\right\rangle \approx\left|x-x^{\prime}\right|^{2 \chi} f\left(\frac{\left|t-t^{\prime}\right|}{\left|x-x^{\prime}\right|^{z}}\right)
$$

Here $f$ is a scaling function and $\chi$ and $z$ are the roughness and dynamic exponents, respectively, with values which depend on the surface dynamics. For an EW interface $\chi=1 / 2, z=2$ while for a KPZ interface $\chi=1 / 2$, $z=3 / 2$.

The hard-core particles slide downwards along the local slope $\left(\frac{\partial h}{\partial x}\right)$ of the interface. In the overdamped limit, their velocity is proportional to the local gradient of height. The equation governing the evolution of particle density can be derived from the continuity equation $\frac{\partial \rho(x, t)}{\partial t}=-\frac{\partial J(x, t)}{\partial x}$. The local current $J(x, t)$ has a systematic part $\rho(1-\rho)\left(1-2 \frac{\partial h}{\partial x}\right)$, a diffusive part $-\nu_{2} \frac{\partial \rho}{\partial x}$ (driven by local density inhomogeneity) and a stochastic part $\eta_{2}(x, t)$ (a Gaussian white noise). The timeevolution equation for the density fluctuation $\tilde{\rho}=\rho-\rho_{0}$ is then

$$
\begin{gathered}
\frac{\partial \tilde{\rho}}{\partial t} \quad=\nu_{2} \frac{\partial^{2} \tilde{\rho}}{\partial x^{2}}+2 \rho_{0}\left(1-\rho_{0}\right) \frac{\partial^{2} h}{\partial x^{2}} \\
-\left(1-2 \rho_{0}-2 \tilde{\rho}\right)\left(\frac{\partial \tilde{\rho}}{\partial x}\right)\left[1-2\left(\frac{\partial h}{\partial x}\right)\right]+ \\
2\left(1-2 \rho_{0}\right) \tilde{\rho} \frac{\partial^{2} h}{\partial x^{2}}-2 \tilde{\rho}^{2} \frac{\partial^{2} h}{\partial x^{2}}+\frac{\partial \eta_{2}(x, t)}{\partial x}
\end{gathered}
$$

We will not analyze this equation directly; rather we will study the particle dynamics by performing numerical simulations on a lattice model, described in section 3 , whose long distance and long time properties are expected to be described by Eqs.(2) and (3).

\section{B. FDPO : Static Properties}

In an earlier study on static properties of this model 7], the density-density correlation $C(r, L)$ of the sliding par- ticles was measured and found to be a scaling function of $r / L$ in the scaling limit $r \rightarrow \infty, L \rightarrow \infty$ with $r / L$ fixed, as for phase-ordered states. The scaling function has a finite intercept $m^{2}$, and for small argument it decays with a cusp:

$$
\begin{aligned}
C(r, L) & =f\left(\frac{r}{L}\right) \\
& =m^{2}\left(1-a\left(\frac{r}{L}\right)^{\alpha}\right), \quad\left(\frac{r}{L} \ll 1\right)
\end{aligned}
$$

$m^{2}$ is a measure of long range order (LRO) as LRO is defined by the large $r$ (scaling limit) behavior of the correlation function. In the limit of an infinite system, this corresponds to $r / L \rightarrow 0$.

The value of the cusp exponent $\alpha$ depends on the dynamics of the driving surface: $\alpha \simeq 0.5$ for particles on an EW interface while $\alpha \simeq 0.25$ when the underlying surface is of KPZ type 77. In a related coarse-grained surface model defined in [7] and discussed in section 3 below, the correlation function was shown analytically to have the above scaling form with $m^{2}=1$ and $\alpha=0.5$ for both EW and KPZ surfaces. For hard-core particles sliding on a two dimensional surface, the same scaling form is found for $C(r, L)$, but with a different value of the intercept and the cusp exponent. For customary phase-ordering systems, it is expected that this scaling function should decay linearly, consistent with the Porod law [13]. The cusp $(\alpha<1)$ is one manifestation of the unusual nature of FDPO.

For a phase-ordered system, the lowest non-zero Fourier component of the density profile measures the extent of phase-separation and is an appropriate order parameter. A characteristic of FDPO is that the distribution of this order parameter remains broad even in the thermodynamic limit, indicating the presence of strong fluctuations.

\section{FDPO : Dynamical Properties}

In this paper, we study the dynamics associated with FDPO. Our results are summarized below. We find that the steady state auto-correlation in the density fluctuation $\sigma(x, t)$ of the sliding particles (SP): $A_{S P}(t)=$ $\langle\sigma(x, 0) \sigma(x, t)\rangle$ is a scaling function of $t / L^{z}$, consistent with the notion of phase ordering. For $t \ll L^{z}$, this scaling function decays with a cusp, in contrast to a linear decay normally expected for phase-ordering systems. The presence of the cusp is the dynamical manifestation of the unconventional character of FDPO.

We also monitored the auto-correlation function in the aging regime $\mathcal{A}_{S P}\left(t_{1}, t_{2}\right)=\left\langle\sigma\left(x, t_{1}\right) \sigma\left(x, t_{1}+t_{2}\right)\right\rangle$. From the dynamic scaling hypothesis, $\mathcal{A}_{S P}\left(t_{1}, t_{2}\right)$ should be a scaling function of $t_{1} / t_{2}$, the ratio of initial time to the time lag [12]. In the limit $t_{2} \gg t_{1}$, the scaling function shows a power law decay with an exponent that depends on the phase-ordering kinetics. In the opposite limit $t_{1} \gg$ $t_{2}$, the scaling function decays with a cusp as in steady 
state, the only difference being that the system size $L$, as appears in the steady state scaling function, is replaced by the coarsening length $t_{1}^{1 / z}$.

The space-time correlation for the sliding particles in steady state, defined as $G_{S P}(r, t)=\langle\sigma(x, 0) \sigma(x+r, t)\rangle$ is a function of $r / L$ and $t / L^{z}$. When plotted against $t / L^{z}$ for a fixed value of $r / L$, this function is non-monotonic. and decays like the steady state auto-correlation for large $t$. The auto-correlation function in steady state and in the aging regime, together with the space-time correlation function in steady state constitute our dynamical characterization of FDPO.

\section{FDPO : An Ordered State?}

The distinctive feature of FDPO is the presence of strong fluctuations in steady state. On the one hand, a diagnostic of LRO such as a non-zero value of the intercept $m^{2}$ indicates an ordered state. On the other hand, the macroscopic state of the system changes relatively rapidly over a time-scale $\sim L^{z}$, in contrast to a normal phase-ordered system, where the typical time grows exponentially with $L$. The small lifetime of a given macroscopic ordered state gives pause, and seems to contradict the notion of LRO. To resolve this apparent contradiction we study the time dependence of the length of the largest particle cluster $l_{\max }(t)$. We conclude that the short lifetime is associated with the system wandering over a multitude of ordered states, each very different from the other, but all characterized by a large value of $l_{\max }(t)$. Dynamical excursions away from this attractor of ordered states are extremely infrequent and associated with an exponentially growing time scale.

\section{DESCRIPTION OF THE MODEL}

We study a discrete model of a fluctuating interface on which hard-core particles slide downwards under gravity, following the local slope of the interface. The 1-d interface of length $L$, consists of discrete surface elements; the slope of the surface elements between the $i$-th and $(i+1)$ th site is $\tau_{i+\frac{1}{2}}$, which can take the value +1 or -1 . Accordingly the height at site $i$ is given by $h_{i}=\sum_{j=1}^{i} \tau_{j-\frac{1}{2}}$. The dynamics of the interface involves stochastic corner flips with exchange of adjacent $\tau$ 's; the transition $/$ to $\backslash$ occurs with a rate $p_{1}$ while $\backslash /$ to $\bigwedge$ with rate $q_{1}$. We have taken $p_{1}=q_{1}=1$ for an EW surface and $p_{1}=1, q_{1}=0$ for KPZ surface. The overall slope $\mathcal{T}=\frac{1}{L} \sum_{i=1}^{L} \tau_{i+\frac{1}{2}}$ is conserved and in our case we will consider $\mathcal{T}=0$, meaning that the interface is untilted.

The hard-core particles are represented by variables $\left\{\sigma_{i}\right\}$ each of which takes a value +1 or -1 according as the $i$-th site contains a particle or a hole. The deviation from half-filling $\mathcal{S}=\frac{1}{L} \sum_{i=1}^{L} \sigma_{i}$ is conserved and we consider $\mathcal{S}=0$, corresponding to the case of half- filling. A particle and hole on adjacent sites $(i, i+1)$ exchange with rates that depend on the intervening local slope $\tau_{i+\frac{1}{2}}$; thus the moves $\bullet \backslash \circ \rightarrow \circ \backslash \bullet$ and $\circ / \bullet \rightarrow \bullet / \circ$ occurs at rate $p_{2}$ while the inverse moves occur at rate $q_{2}$. In the case when the particles are sliding downwards along gravity, we have $q_{2}<p_{2}$. We have considered $p_{2}=1$ and $q_{2}=0$. Because of the hard-core exclusion between the particles, with the above update rules, the system has a particle-hole symmetry, i.e. any correlation function involving the density variable remains invariant as the particle density is replaced by the hole density. This implies that unlike the case of non-interacting particles where the correlation function of the density variable is qualitatively different between the advection case and the anti-advection case [5], the two processes show identical correlation behavior here.

From the dynamical rules, it follows that the movement of particles depends on the fluctuations of the underlying interface. Due to gravity the particles tend to slide down into local valleys. However, in the non-equilibrium system under consideration, before the particles can fill in the lowest valley, the interface evolves, often causing the valley to turn over. Nevertheless, it is useful to consider the adiabatic limit where the interface moves infinitely more slowly than the particles, in which case the particles have ample time to explore the landscape and eventually settle in the deepest valleys. It seems plausible that the dynamics of hills and valleys of the interface may provide insight into the dynamics of the particles. This motivates the definition of a coarse-grained depth model (CD model) as follows []. Consider the variable $s_{i}(t)$ defined as $s_{i}(t)=\operatorname{sgn}\left[h_{i}(t)-\langle h(t)\rangle\right]$, where $\langle h(t)\rangle$ is the average height at time $t:\langle h(t)\rangle=\frac{1}{L} \sum_{i=1}^{L} h_{i}(t)$. The variable $s_{i}(t)$ can take values $+1,-1$ or 0 , depending on whether the position of the $i$-th site is above, below or at the average level. In other words, $s_{i}(t)$ gives a coarse-grained description of the surface by labeling 'highlands' and 'lowlands'. For an EW interface, the dynamics is tractable and we obtain an analytic expression for time-dependent correlations of $s_{i}(t)$. These results might be expected to be close to those of $\sigma_{i}(t)$ in the extreme adiabatic limit. As a matter of fact, we find that they also describe qualitatively the particle model even in the strongly non-equilibrium case.

\section{AUTO-CORRELATION FUNCTION IN STEADY STATE}

We have studied the auto-correlation $A(t, L)$ involving the density variable $\left\langle\sigma_{i}(0) \sigma_{i}(t)\right\rangle$ in the sliding particle (SP) model and also that for the CD variables $\left\langle s_{i}(0) s_{i}(t)\right\rangle$ in the CD model. Periodic boundary conditions are used. We will see below that in the steady state of a system of size $L$, the auto-correlation $A(t, L)$ is a scaling function of $\frac{t}{L^{z}}$, where $z$ is the dynamic exponent defined earlier. This scaling function shows a cusp in the small argument limit, as seen previously in the static correlation scaling 


\begin{tabular}{|c||c|c||c|c|}
\hline \multicolumn{1}{|c||}{} & \multicolumn{2}{c|}{ CD Model } & \multicolumn{2}{c|}{ SP Model } \\
\cline { 2 - 5 } & EW & KPZ & EW & KPZ \\
\hline \hline$m$ & 1.0 & 1.0 & $0.82 \pm 0.03$ & $0.75 \pm 0.04$ \\
\hline$\beta^{\prime}$ & 0.25 & $0.31 \pm 0.02$ & $0.22 \pm 0.02$ & $0.18 \pm 0.01$ \\
\hline$\lambda$ & 0.5 & 0.5 & $0.26 \pm 0.005$ & $0.15 \pm 0.005$ \\
\hline$\gamma$ & 0.75 & $0.84 \pm 0.02$ & $0.69 \pm 0.02$ & $0.82 \pm 0.04$ \\
\hline
\end{tabular}

TABLE I: The values of relevant exponents and order parameter for dynamical characterization of CD model and SP model

function [Eq. (5)]:

$$
\begin{aligned}
A(t, L) & =h\left(\frac{t}{L^{z}}\right) \\
& =m^{2}\left[1-b\left(\frac{t}{L^{z}}\right)^{\beta^{\prime}}\right], \quad \frac{t}{L^{z}} \rightarrow 0
\end{aligned}
$$

$m$ is a measure of the LRO as explained in section 2. Note that for large enough time, the auto-correlation function is expected to factorize and become equal to $\mathrm{m}^{2}$.

However, for small time, $t \lesssim 1$, which falls outside the scaling regime, the auto-correlation function shows a linear drop with an $L$-dependent slope:

$$
A(t, L) \approx 1-b^{\prime} \frac{t}{L^{\lambda}}, \quad(t \lesssim 1)
$$

If $m^{2}=1$, as shown below for the CD model, matching Eqs.(7) and (8) for $t \simeq 1$ yields

$$
\lambda=z \beta^{\prime}
$$

If $m^{2} \neq 1$, as happens for the SP model, a relation between the exponents cannot be obtained. Instead, the matching condition determines a time scale $t^{*}$ for the crossover from the linear decay in Eq. (8) to the cuspy decay in Eq. (17). In the large $L$ limit, we find to the leading order,

$$
t^{*}=\frac{1-m^{2}}{b^{\prime}} L^{\lambda}
$$

Let us illustrate these properties, by discussing the auto-correlation in the CD model, defined as $A_{C D}(t, L)=$ $\left\langle s_{i}(0) s_{i}(t)\right\rangle$. First consider short times $t \lesssim 1$. At $t=0$ let the initial configuration of the surface be $\left\{h_{i}(0)\right\}$. As time passes, there are stochastic corner flips, as described in section 3. However, only those flips occurring close to the average level can cause a change in the CD variable $s_{i}(t)$, as any local fluctuation far above or below the average level, would not change the sign of $s_{i}(t)=\left(h_{i}(t)-\langle h(t)\rangle\right)$. More precisely, only those sites in $\left\{h_{i}(0)\right\}$ which have at least one neighbor situated exactly on the average level, putatively contribute to the drop in auto-correlation function. Now, for a self-affine surface of length $L$ and roughness exponent $\chi$, the number of such points scales as $L^{1-\chi}$ and the density of such points goes as $L^{-\chi}$ [14]. For small $t$, the probability that any one of these points will actually take part in a local fluctuation is proportional to $t$. This immediately implies $A_{C D}(t \lesssim 1, L) \approx 1-b_{1}{ }^{\prime} \frac{t}{L^{x}}$. Comparison with Eq. (8) shows that for the CD model, we have $\lambda=\chi=\frac{1}{2}$. Note that although EW and KPZ surfaces have different dynamics, the above argument holds for both of them as their stationary measure is the same in 1-d.

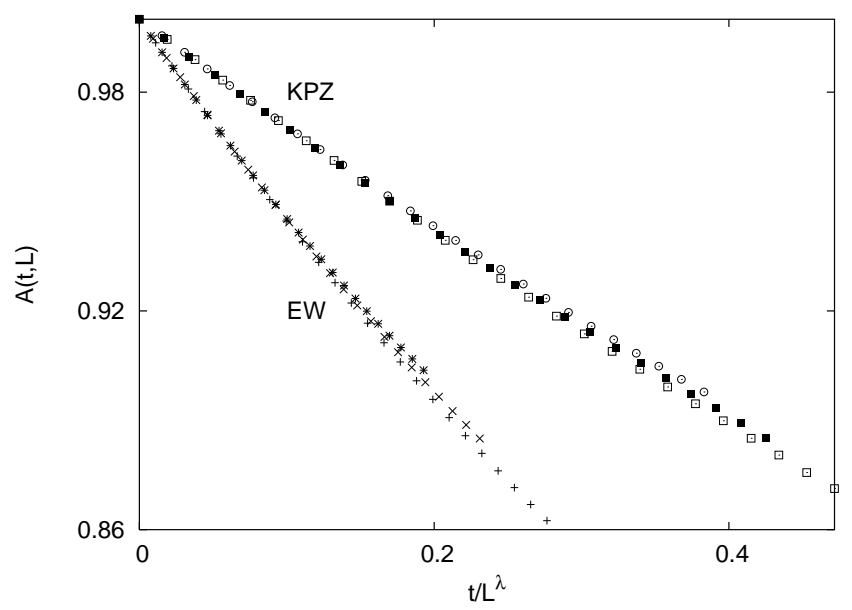

FIG. 1: Illustrating the linear drop of $A(t, L)$ for short times $t \lesssim 1$ in the $S P$ model for system size $L=128,256,512$.

For the particle model, although the initial drop is found to be linear as described in Eq. (8), the exponent $\lambda$ takes the value $0.26 \pm 0.005$ for particles on an EW surface and $0.15 \pm 0.005$ for particles on a KPZ surface. The data are shown in Fig.(11).

For $t \geq 1$, we have analytically calculated $A_{C D}(t, L)$ for an EW interface. This exploits the fact that $h_{i}(t)$ in this case is a Gaussian variable, implying $s_{i}$ correlations satisfy the arc-sine law

$$
\left\langle s_{i}(t) s_{i}(0)\right\rangle=\frac{2}{\pi} \sin ^{-1}\left(\frac{\left\langle H_{i}(t) H_{i}(0)\right\rangle}{\sqrt{\left\langle H_{i}^{2}(t)\right\rangle\left\langle H_{i}^{2}(0)\right\rangle}}\right)
$$

where $H_{i}(t)=h_{i}(t)-\langle h(t)\rangle$, which is also a Gaussian variable. If $\tilde{h}_{k}(t)$ is the Fourier transform of $h_{i}(t)$, the numerator in the argument of arcsine can be written as $\sum_{k \neq 0}\left\langle\tilde{h}_{k}(t) \tilde{h}_{-k}(0)\right\rangle=\sum_{k \neq 0} \Gamma \frac{\exp \left(-c_{k} t\right)}{c_{k}}$, using the discrete version of the EW equation. Here, $c_{k}=4 \nu_{1} \sin ^{2} \frac{k}{2}$. Moreover, $\left\langle H_{i}{ }^{2}(t)\right\rangle=\left\langle H_{i}{ }^{2}(0)\right\rangle=\Gamma \sum_{k \neq 0} \frac{1}{c_{k}}$. Thus we have

$$
\left\langle s_{i}(t) s_{i}(0)\right\rangle=\frac{2}{\pi} \sin ^{-1}\left[\frac{\sum_{k \neq 0} \frac{\exp \left(-c_{k} t\right)}{c_{k}}}{\sum_{k \neq 0} \frac{1}{c_{k}}}\right]
$$

We have numerically evaluated this discrete sum and plotted it in Fig.(2a) against the scaling argument $t / L^{2}$ for different $L$ values. The cusp exponent can be read off from the plot in the inset. 
In the continuum limit, Eq.(12) becomes

$$
\langle s(x, t) s(x, 0)\rangle=\frac{2}{\pi} \sin ^{-1}\left[\frac{\int_{\frac{2 \pi}{L}}^{\pi} d k \frac{\exp \left(-k^{2} t\right)}{k^{2}}}{\int_{\frac{2 \pi}{L}}^{\pi} \frac{d k}{k^{2}}}\right]
$$

The integral in the numerator takes the form $\frac{L \Gamma}{\pi}\left[\frac{L}{2 \pi} \exp \left(-\frac{4 \pi^{2} t}{L^{2}}\right)+\sqrt{\pi t} \operatorname{erf}\left(\frac{2 \pi \sqrt{t}}{L}\right)-\sqrt{\pi t}\right]$. In the limit $t / L^{2} \ll 1$, this becomes, to the leading order, $\frac{L \Gamma}{\pi}\left[\frac{L}{2 \pi}-\sqrt{\pi t}\right]$. Noting that the denominator is $\frac{L \Gamma}{\pi} \cdot \frac{L}{2 \pi}$ and expanding for small values of $\frac{\sqrt{t}}{L}$, we get

$$
\langle s(x, t) s(x, 0)\rangle \approx 1-\frac{4}{\pi^{\frac{1}{4}}}\left(\frac{t}{L^{2}}\right)^{1 / 4} \quad\left(t / L^{2} \ll 1\right)
$$

Comparing with Eq.(7) gives $m^{2}=1, \beta^{\prime}=\frac{1}{4}, z=2$.

For the KPZ surface, the time evolution equation for the height field is not Gaussian and hence such an analytical treatment is not possible. We study $A_{C D}(t)$ using Monte Carlo simulation. No initial equilibration is required as the steady state measure for a KPZ surface with periodic boundary conditions gives equal weight to every configuration. We followed the update rules discussed in section 2 and averaged over sites as well as over $10^{5}$ histories. The results are shown in Fig.(2b). A good scaling collapse is obtained for different $L$, on rescaling the time to $t / L^{z}$ with $z=\frac{3}{2}$. The cusp exponent $\beta^{\prime}$ was extracted by plotting $m^{2}-A_{C D}(t)$ against $t / L^{z}$ (shown in the inset), using $m^{2}=1$ and this gives $\beta^{\prime}$ to be $0.31 \pm 0.02$. Our best estimate corresponds to the largest system size $L=2048$. The error-bar is based on the values of $\beta^{\prime}$ obtained for smaller system size $(L=512,1024)$; the statistical error is much smaller.

For the sliding particle (SP) model, the steady state measure is not known analytically. In our simulation, we started from a randomly disordered configuration and allowed a long time $\sim 10 L^{z}$ for the system to reach a steady state. We then measured $\frac{1}{L} \sum_{i=1}^{L} \sigma_{i}(0) \sigma_{i}(t)$ for approximately $L^{z}$ time-steps. We waited several thousand timesteps before repeating the procedure, and averaged over $10^{4}$ histories .

For particles sliding on an EW interface, we obtained a good scaling collapse of $A_{S P}(t, L)$ for different $L$ after rescaling time to $t / L^{2}$ [Fig.(2a)]. The cusp exponent was extracted by fitting $m^{2}-A_{S P}(t, L)$ to a power law. We have estimated $m^{2}$ by using the same technique as discussed in [7]. The best estimate of $m^{2}$ corresponds to the value for which the structure factor has the largest power law stretch. We found that $m^{2}$ shows a systematic dependence on $L$ and the cusp exponent $\beta^{\prime}$ is in fact quite sensitive to the value of $m^{2}$. We have used $m^{2} \simeq 0.82$, our estimate from the largest system size we could access $(L=4096)$. This yields $\beta^{\prime} \simeq 0.22$. On the other hand, using $m_{\infty}^{2} \simeq 0.85$, which we get by extrapolating the dependence of $m^{2}$ on $L$ for an infinite system, we find $\beta^{\prime} \simeq 0.20$.

For the SP model on a KPZ surface, we find $m^{2} \simeq$ 0.75 . Figure $(2 \mathrm{~b})$ shows the scaling collapse for different
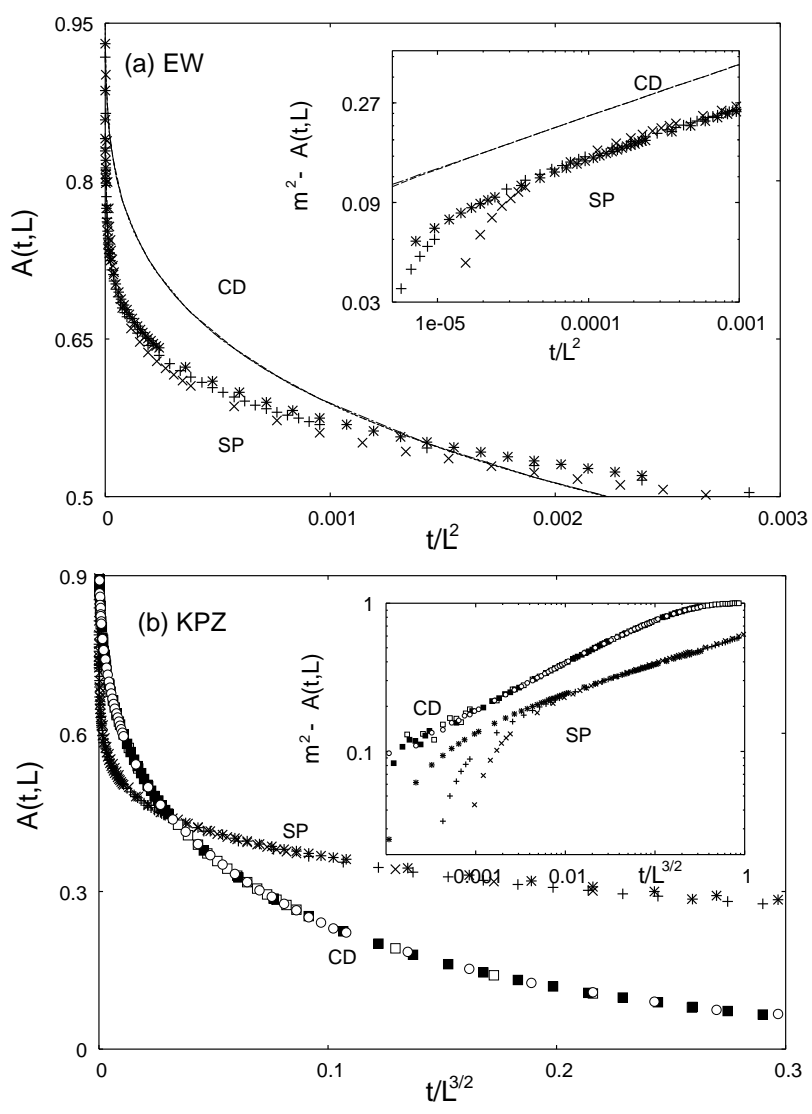

FIG. 2: Scaled auto-correlation function in steady state for $S P$ and $C D$ models for (a) $E W$ and (b) KPZ interfaces. In both cases, we used $L=512,1024,2048$. The cusp exponents were estimated using the plots shown in the inset.

$L$ after rescaling the time by $L^{3 / 2}$. The inset shows that $m^{2}-A_{S P}(t, L)$ follows a power law and the exponent is found to be $\beta^{\prime} \simeq 0.18$. The value of $\beta^{\prime}$ obtained using $m_{\infty}^{2}$ is $\simeq 0.17$.

\section{AUTO-CORRELATION IN AGING REGIME}

The aging auto-correlation function $\mathcal{A}\left(t_{1}, t_{2}\right)$ is defined as $\left\langle\sigma_{i}\left(t_{1}\right) \sigma_{i}\left(t_{1}+t_{2}\right)\right\rangle$ for the particles and as $\left\langle s_{i}\left(t_{1}\right) s_{i}\left(t_{1}+\right.\right.$ $\left.\left.t_{2}\right)\right\rangle$ for the CD variables. $\mathcal{A}\left(t_{1}, t_{2}\right)$ depends on both $t_{1}$ and $t_{2}$. For $1 \ll t_{1}, t_{2} \ll L^{z}, \mathcal{A}\left(t_{1}, t_{2}\right)$ is a function of $\frac{t_{1}}{t_{2}}$, as expected for phase ordering systems [12]. In the limit when $t_{2} \gg t_{1}$, this scaling function has a power law decay

$$
\mathcal{A}\left(t_{1}, t_{2}\right) \sim\left(\frac{t_{1}}{t_{2}}\right)^{\gamma} \text { for } t_{2} \gg t_{1}
$$

while in the opposite limit, $t_{1} \gg t_{2}$, the scaling function has the form

$$
\mathcal{A}\left(t_{1}, t_{2}\right) \sim m^{2}\left(1-b_{1}\left(\frac{t_{2}}{t_{1}}\right)^{\beta^{\prime}}\right) \text { for } \frac{t_{2}}{t_{1}} \rightarrow 0
$$


This is similar to the form of the steady-state autocorrelation in Eq. (77) with $L$ replaced by $t_{1}^{1 / z}$, meaning that locally the system has reached steady state over a length scale of $t_{1}^{1 / z}$.
We first present our results on the CD model. As in the case of steady-state auto-correlation, we have been able to calculate $\mathcal{A}_{C D}\left(t_{1}, t_{2}\right)$ for an EW surface analytically. Following similar steps as in the last section, we obtain

$$
\mathcal{A}_{C D}\left(t_{1}, t_{2}\right)=\frac{2}{\pi} \sin ^{-1}\left[\frac{\sum_{k \neq 0} \frac{\exp \left(-c_{k} t_{2}\right)-\exp \left[-c_{k}\left(2 t_{1}+t_{2}\right)\right]}{c_{k}}}{\left\{\sum_{k^{\prime} \neq 0} \frac{1-\exp \left(-2 c_{k^{\prime}} t_{1}\right)}{c_{k^{\prime}}}\right\}^{1 / 2}\left\{\sum_{k^{\prime \prime} \neq 0} \frac{1-\exp \left[-2 c_{k^{\prime \prime}}\left(t_{1}+t_{2}\right)\right]}{c_{k^{\prime \prime}}}\right\}^{1 / 2}}\right]
$$

Taking the continuum limit and using $t_{1}, t_{2} \ll L^{2}$, we obtain

$$
\mathcal{A}_{C D}\left(t_{1}, t_{2}\right)=\frac{2}{\pi} \sin ^{-1}\left[\frac{\sqrt{2 t_{1}+t_{2}}-\sqrt{t_{2}}}{\left(2 t_{1}\right)^{1 / 4}\left(2 t_{1}+2 t_{2}\right)^{1 / 4}}\right]
$$

In the limit $t_{2} \gg t_{1}$, right hand side becomes $\frac{\sqrt{2}}{\pi}\left(\frac{t_{1}}{t_{2}}\right)^{3 / 4}$. Comparing with Eq.(15), we get $\gamma=\frac{3}{4}$. In the opposite limit, when $t_{1} \gg t_{2}$, the right hand side becomes, after simplification,

$$
\mathcal{A}_{C D}\left(t_{1}, t_{2}\right) \approx 1-\frac{2^{\frac{5}{4}}}{\pi}\left(\frac{t_{2}}{t_{1}}\right)^{1 / 4}
$$

Comparing with Eq.(16), we find $\beta^{\prime}=1 / 4$, as expected.

Figure (3a) shows the numerical evaluation of the discrete sum in Eq. (17). The power law characterizing the decay has been shown in the inset.

In our Monte Carlo simulations, we have a spatial average as well as an average over $10^{4}$ histories. For the CD model of a KPZ surface, we started with a flat interface as an initial condition and evolved it in time to measure $\mathcal{A}_{C D}\left(t_{1}, t_{2}\right)$. The results are shown in fig.(3b). The best estimate of the cusp exponent corresponds to $t_{1}=32000$ and the error bar is based on its values for $t_{1}=2000,8000$. This finally gives $\beta^{\prime}=0.31 \pm 0.01$, which is close to the steady state value. The inset shows the power law decay and the exponent $\gamma$ takes the value $0.84 \pm 0.03$. Here, the best estimate is for $t_{1}=500$ and the error-bar is for $t_{1}=2000,8000$.

For the SP model on an EW interface, we start with randomly distributed particles on a random surface profile. The aging auto-correlation $\mathcal{A}_{S P}\left(t_{1}, t_{2}\right)$ shows a scaling collapse as plotted against $t_{2} / t_{1}$ [see fig.(3a)]. The value of the cusp exponent $\beta^{\prime}$ is $0.20 \pm 0.02$, close to its steady state value. The inset shows plot in the regime $t_{2} \gg t_{1}$. The power law exponent in this case is $\gamma=0.69 \pm 0.02$.

The SP model on a KPZ surface also starts with the random initial condition. The data are shown in fig.(3b). The exponents are $\beta^{\prime}=0.17 \pm 0.01$ and $\gamma=0.82 \pm 0.04$.

\section{SPACE-TIME CORRELATION IN STEADY STATE}

In this section, we discuss the behavior of spacetime correlation $G(r, t, L)$ defined in steady state as $\left\langle\sigma_{i}(0) \sigma_{i+r}(t)\right\rangle$ for the particles and $\left\langle s_{i}(0) s_{i+r}(t)\right\rangle$ for the CD variable. $G(r, t, L)$ does not show any $L$-independent scaling between $r$ and $t$. Rather, it is a function of the scaled variables $\xi=r / L$ and $\tau=t / L^{z}$

$$
G(r, t, L)=g(\xi, \tau)
$$

With $\xi$ held fixed, $g$ shows an interesting non-monotonic behavior with $\tau . g(\xi, 0)$ reduces to the pair correlation function $f(\xi)$ [see Eq. (44)], and as $\tau$ increases, $g(\xi, \tau)$ is observed to rise and attain a peak [see Fig.(4a)]. Finally for larger $\tau$, it decreases and merges with the autocorrelation scaling function $h(\tau)$ [see Eq.(6)]. Note that $G(r=0, t, L) \equiv A(t, L)$, and by continuity, we expect that when $\xi^{z} \ll \tau$, the scaling function should behave like $h(\tau)$. From our knowledge of the scaling functions $f(\xi)$ and $h(\tau)$, we have been able to verify that $f(\xi)<h\left(\tau=\xi^{z}\right)$. This implies that $g(\xi, \tau)$ must show an initial rise.

For the CD model on an EW interface,

$$
G_{C D}(r, t, L)=\frac{2}{\pi} \sin ^{-1}\left[\frac{\sum_{k>0} \frac{\exp \left(-c_{k} t\right) 2 \cos (k r)}{c_{k}}}{\sum_{k>0} \frac{1}{c_{k}}}\right] .
$$

We have evaluated this sum numerically and plotted it against $\tau$, for a fixed value of $\xi$ in Fig.(4a), inset which shows its non-monotonic nature. In the continuum limit, the argument of arcsine takes the form $\quad\left[2 \cos (2 \pi \xi)-2 \pi^{2} \xi+2 \pi \xi S i(2 \pi \xi)-2 \pi \nu_{1} N(\xi, \tau)\right]$ where $N(\xi, \tau)$ is defined as $\int_{0}^{\tau} d y \sqrt{\frac{\pi}{\nu_{1} y}} \exp \left(-\frac{\xi^{2}}{4 \nu_{1} y}\right)\left[\operatorname{erf}\left(2 \pi \sqrt{\nu_{1} y}-\frac{i \xi}{2 \sqrt{\nu_{1} y}}\right)-1\right]$ which shows explicitly that $G_{C D}(r, t, L)$ is a function of $\xi$ and $\tau$ only.

To measure $G_{S P}(r, t, L)$ for particles on an EW surface we performed Monte Carlo simulations as before. After equilibrating the system, we measure $\frac{1}{L} \sum_{i=1}^{L} \sigma_{i}(0) \sigma_{i+r}(t)$ for about $L^{z} / 10$ time steps, then after a gap of a few hundred time steps, we take another 

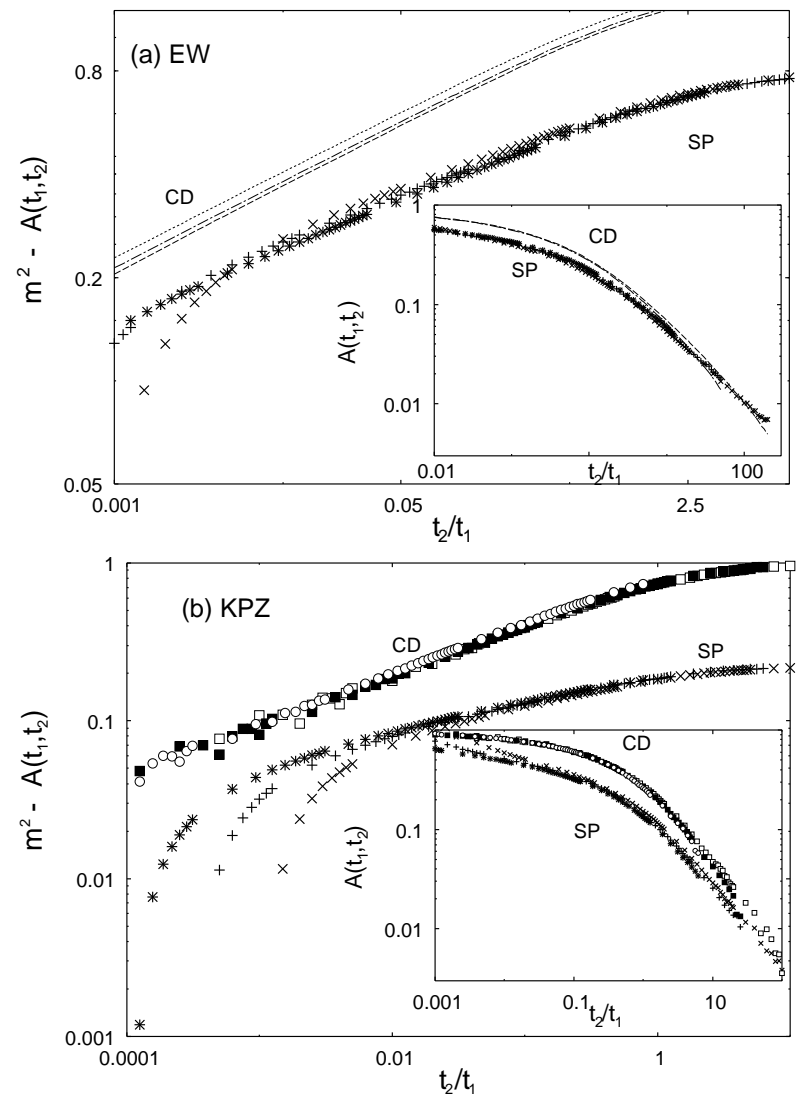

FIG. 3: Aging auto-correlation for $C D$ and $S P$ models with (a) EW and (b) KPZ interfaces. The cusp exponent $\beta^{\prime}$ was determined for $t_{1} \gg t_{2}$, after subtraction from $m^{2}$. The $C D$ model data in (a) has been multiplied by 1.5 to distinguish it from the SP model data points. The inset shows the power law behavior in the regime $t_{1} \ll t_{2}$. We used $L=2048$ in (a) and $L=8192$ in (b). The Inset shows the data with $t_{1}=500,2000,8000$ in both (a) and (b). For extraction of $\beta^{\prime}$, we used $t_{1}=2000,8000,32000$.

set of data. We finally average over $10^{5}$ such histories. The results are shown in Fig.(4a) where we have also included the scaling function $h(\tau)$ to compare the long time behavior. The corresponding results for KPZ surface are shown in Fig.(4b).

\section{LARGEST CLUSTER IN STEADY STATE}

One of the key characteristics of FDPO is the presence of strong fluctuations, even in the thermodynamic limit. In the steady state, large clusters are present in the system and the cluster size distribution follows a power law. As a result of fluctuations, these clusters undergo large changes in their lengths, associated with the fact that the macroscopic state of the system keeps changing. For a system of size $L$, the typical lifetime of a macrostate scales as $L^{z}$. The question arises: if the lifetime of a state is so much smaller than exponential, in what sense can we call such a state a 'phase'? We have addressed this
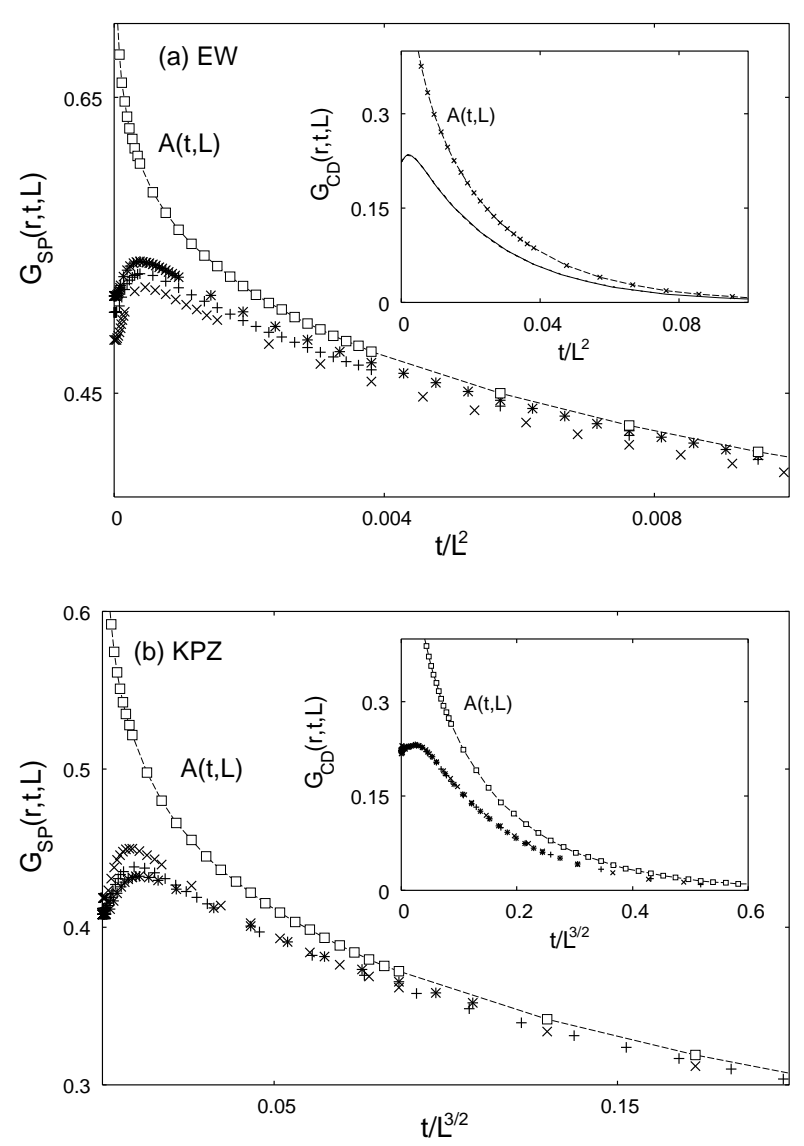

FIG. 4: The time dependence of $G(r, t, L)$ is shown for particles on an (a)EW and (b) KPZ surface for $\frac{r}{L}=0.016$. The values of $L$ are 256, 512, 1024 for (a) and 512, 1024, 2048 for (b). The scaled auto-correlation is also shown, for comparison. The insets show the same quantity calculated for the corresponding CD model with $\frac{r}{L}=0.125$ for both cases.

question in the following way. Let $l_{\max }(t)$ be the length of the largest cluster present in the system at time $t$. In a disordered state, this length scales as $\log L$. But starting from a random initial configuration, as the system approaches steady state, $l_{\max }(t)$, although a fluctuating quantity, shows an increasing trend. Finally, in steady state, $l_{\max }(t)$ is still fluctuating, thereby changing the macroscopic state of the system. But $l_{\max }(t)$ continues to remain substantially above its disordered state value $\log L$. In other words, the system manages to retain its ordered character despite steady state fluctuations. The system continues to move from one macroscopic state to other over a time-scale of $L^{z}$. But each of these states are ordered in the sense that they all correspond to large values of $l_{\max }(t)$.

We have studied the distribution of $l_{\max }$ in steady state as well as in disordered state. Our studies show that as system size increases, the overlap between these two distributions falls off. This means that as $L$ grows, it is increasingly unlikely for the steady state $l_{\max }$ to come down as low as its disordered state value. The time-scale 
for such a transition in fact grows exponentially with $L$.

The disordered state distribution was obtained by averaging over $10^{8}$ data points. The mean of this distribution scales as $\log L$ as mentioned earlier.

After the system has reached steady state, we measure the largest cluster present in that configuration. We let the configuration evolve in time and after waiting for few hundred time steps, we again measure $l_{\max }(t)$. We obtain the distribution $P\left(l_{\max }, L\right)$ after normalizing over $10^{6}$ such data points. As shown in the following figures, $P\left(l_{\max }, L\right)$ for different $L$ values undergo a scaling collapse as $l_{\max }$ is rescaled by the mean of the distribution $\langle l\rangle$. We have found that $\langle l\rangle \sim L^{\phi}$, where the exponent $\phi$ depends on the dynamical rules. For particles on an EW surface $\phi \simeq 0.86$, whereas for $\mathrm{KPZ}$ advection, $\phi \simeq 0.60$ while for $\mathrm{KPZ}$ anti-advection $\phi \simeq 0.91$. We show the data for KPZ advection in fig.(15).

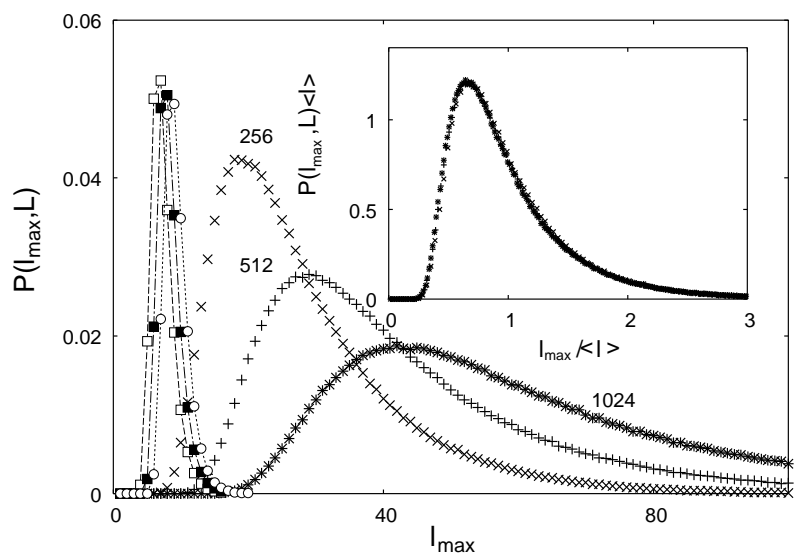

FIG. 5: The distribution of the length of the largest cluster $P\left(l_{\max }, L\right)$ for particles advected by KPZ surface is shown for $L=256,512,1024$, with the scaling collapse in the inset.The curves to the left show the same distribution in disordered state, after rescaling the $y$-axis by 0.2

\section{DISCUSSION}

In this paper, we have studied the dynamics of interacting passive scalars driven by a fluctuating EdwardsWilkinson or Kardar-Parisi-Zhang surface (or equivalently, a Burgers fluid), by characterizing the scaling properties of correlation functions in steady state and those of aging correlations during the approach to steady state. It is instructive to compare our results with ear- lier work on the dynamics of passive scalars in different contexts.

Mitra and Pandit 9] studied the dynamical properties of a system of non-interacting passive particles, advected by an incompressible fluid, whose velocity field is drawn from the Kraichnan ensemble, and therefore has power law correlations in space, but is delta-correlated in time. By contrast, we have studied passive particles with hardcore interactions, advected (in the Burgers case) by a compressible flow which has power law correlations in time. The compressible nature of the flow results in particles being driven together in our case. The resulting state is described by a space-time correlation function $G(r, t, L)$ which is a function of the scaling combinations $r / L$ and $t / L^{z}$, as in any phase-ordering system. However, there is no non-trivial $r-t$ scaling in the limit $L \rightarrow \infty$. On the contrary, in [9], $G(r, t, L)$ was found to show a scaling between $r$ and $t$, with $t \sim r^{z}$ for fixed $L$. This difference of behavior is presumably a reflection of the strong differences between passive scalars with clustering or phase-ordering tendencies, and those which spread out in space.

Even when the driving fluid is compressible, the degree of clustering of the passive particles depends on the nature of interactions between them. In the presence of hard-core interactions, the system reaches a phaseordered state, albeit one with strong fluctuations. As a consequence, in the limit of small scaling argument, the spatial and temporal correlation functions show a cuspy approach to a finite intercept. However, in the absence of any interaction, the passive particles go into a more strongly clustered state, where the correlation functions show a power law divergence at the origin [5].

Finally, the study of the largest cluster allows us to arrive at a simple picture of a fluctuation dominated phase-ordered state. Despite the presence of strong fluctuations, the system never loses its ordered character. Fluctuations carry the system from one ordered configuration to another macroscopically distinct one, over a time-scale $\sim L^{z}$. However, the probability for the system to leave this attractor of ordered states vanishes exponentially with the system size.

\section{ACKNOWLEDGMENTS}

We acknowledge useful discussions with S.N. Majumdar and S.Ramaswamy. SC would like to thank TIFR Endowment Fund for partial financial support.
[1] B.I.Shraiman and E.D.Siggia Nature 405, 369 (2000).

[2] E.Balkovsky, G.Falkovich and A.Fouxon Phys. Rev. Lett. 86, 2790 (2000).

[3] K.Gawedzki and M.Vergassola Physica D 138, 63 (2000).

[4] E.Medina, T.Hwa, M.Kardar, Y.-C.Zhang Phys. Rev. A
39, 3053 (1989).

[5] A.Nagar, M.Barma and S.N.Majumdar Phys. Rev. Lett 94, 240601 (2005).

[6] B.Drossel and M.Kardar Phys. Rev. B 66, 195414 (2002)

[7] D.Das, M.Barma and S.N.Majumdar Phys. Rev. E 64, 
046126 (2001).

[8] G.Manoj and M.Barma J. Stat. Phys. 110, 1305 (2003).

[9] D.Mitra and R.Pandit nlin.CD/0412013

[10] S.F.Edwards, D.R.Wilkinson Proc. R. Soc. London A 381, 17 (1982).

[11] A.-L.Barabási and H.E.Stanley, Fractal Concepts in Surface Growth (Cambridge University Press, New York,1995).
[12] A.J.Bray Adv. Phys. 43, 357 (1994).

[13] G.Porod, in Small Angle X-ray Scattering, edited by O.Glatter and L.Kratky (Academic Press, New York, 1983).

[14] P.Meakin, Fractals,Scaling and Growth Far from Equilibrium (Cambridge University Press, Cambridge,1998) 